\title{
Psychological factors of healthful diet promotion among diabetics: an application of health action process approach
}

\author{
Hosein Rohani ${ }^{1}$, Mohammad Bidkhori ${ }^{2}$, Ahmad Ali Eslami ${ }^{3}$, Erfan Sadeghi ${ }^{4}$, Ahmad Sadeghi ${ }^{5}$
}

\begin{abstract}
${ }^{1}$ Ph.D. of Health Education and Promotion, Assistant Professor, Student Research Committee, Esfarayen Faculty of Medical Sciences, Esfarayen, Iran

${ }^{2}$ M.Sc. of Epidemiology, Department of Public health, Neyshabur University of Medical Sciences, Neyshabur, Iran

${ }^{3} \mathrm{Ph}$.D. of Health Education, Associate Professor, Isfahan University of Medical Sciences, Isfahan, Iran

${ }^{4}$ Department of Biostatistics and Epidemiology, Kermanshah University of Medical Sciences, Kermanshah, Iran

${ }^{5}$ Ph.D. of Health Services Management, Assistant Professor, Department of Public Health, Esfarayen Faculty of Medical Sciences, Esfarayen, Iran
\end{abstract}

\section{Type of article: Original}

\begin{abstract}
Background and aim: Worldwide, type 2 diabetes mellitus (T2DM) prevalence has increased. Non-healthful diet is one of the main reasons to T2DM growth. This study aims to determine the efficacy of the health action process approach (HAPA) in explaining healthful diet (HD) in T2DM patients.

Methods: This cross-sectional study was conducted based on HAPA in Isfahan, Iran between July and December 2015. Participants were recruited by convenience sampling method. HAPA inventory was administered to 203 Isfahan diabetics. Intentions, outcome expectancies, risk perception, action self-efficacy, action and coping planning, maintenance and recovery self-efficacy, and dietary style were the constructs measured. Structural equation modeling (SEM) in AMOS 21 was used to test the hypothesis.

Results: The results based on the common fit indices revealed that the HAPA fitted the data acceptably well among the T2DM patients and within dietary behavior $(\mathrm{p}<0.001)$, RMSEA $=0.082$, CFI $=0.903$, NFI $=0.899)$. Action self-efficacy was the best predictor of intention $(\beta=2.49, \mathrm{p}<0.001)$. Result revealed action and coping planning $(\beta=5.36, \mathrm{p}=0.027)$ and recovery self-efficacy $(\beta=5.67, \mathrm{p}=0.021)$ significantly predicted behavior.

Conclusions: Our findings provide preliminary support for the practical usage of the HAPA model for predicting HD intention and behavior among T2DM patients. Randomized controlled trials should be performed in the future in order to involve causality.

Keywords: Diabetes mellitus; Diet; Health; Health action process approach
\end{abstract}

\section{Introduction}

$\mathrm{T} 2 \mathrm{DM}$ is one of the fastest rising non-communicable diseases in the world (1), which has a vast effect on people and health systems (2). At least 6 million Iranians have T2DM, and its prevalence is increasing, especially among T2DM patients of Isfahan (3-5). Non-healthful diet is one of the main reasons to T2DM growth (4). Large, high-quality clinical trials express that relatively modest changes in diet reduce the prevalence of type 2 diabetes by $50 \%$ for persons with T2DM (6-8). A solution to prevent or to delay the long term effects in patients with T2DM is to manage dietary behaviors (9). However, they are usually exhorted to adopt a healthful diet (HD) behavior which requires changing the food habits, beliefs and meal patterns for their whole lives. Healthful diet is a diet that reminds us of the foods that we should eat more of (vegetables, fruits and whole grain bread and grains) and those we should eat less (meat, cheese, foods with high fat and those with high sugar such as pies, cakes and pastries). This diet is arranged in a way so we eat more fruit, vegetables and grains and less sugar and fat (10). In this regard, educational interventions can be effective in changing T2DM beliefs with respect to HD (11). For this purpose the identification of psychosocial factors underlying dietary behaviors in T2DM patients can be helpful for guiding more effective

\section{Corresponding author:}

Assistant Professor Dr. Hosein Rohani.

Tel: +98.5837241603, Fax: +98.5837238757, Email: hoseinrohani3@gmail.com

Received: May 09, 2017, Accepted: March 01, 2018, Published: April 2018

iThenticate screening: January 12, 2018, English editing: March 05, 2018, Quality control: April 10, 2018

This article has been reviewed / commented by four experts

(C) 2018 The Authors. This is an open access article under the terms of the Creative Commons Attribution-NonCommercialNoDerivs License, which permits use and distribution in any medium, provided the original work is properly cited, the use is non-commercial and no modifications or adaptations are made. 
interventions in these patients. HAPA is a social cognitive framework which has been used in numerous surveys to identify psychological determinants of health behaviors (12-14), and can be used as an educational framework for changing dietary behaviors of T2DM. This model includes the following phases: Motivational and volitional (15). In the former, intentions to implement behavior are affected by the following factors: risk perception, action selfefficacy and outcome expectancies. Risk perception is one's feeling of being at risk for a special health condition which might also motivate him to start changing his health behaviors. Outcome expectancy pertains to perception of benefits gained by adopting the behavior. Therefore, understanding the advantages of performing the HD behavior, will influence intentions to implement the behavior. Action self-efficacy is an individual's perceived ability to implement the new behavior. The development of intentions has more chance to happen in the presence of high action self-efficacy (16). In the volitional phase, the action planning is added in the model as the linkage between intention and behavior. In the context of HD, greater intentions to eating a healthful diet would lead to an increasing chance of making action plans (how, where or when to eat healthful diet) and coping plans (including anticipating obstacles that might hinder the execution of the intended changes, in addition to strategies for dealing with such barriers), thus there will be a higher likelihood for adopting an HD. The HAPA model suggests that an HD, as well as depending on action and coping planning, also depends on perceived ability in adhering to the behavior (maintenance self-efficacy) and in coping after ending the behavior (recovery self-efficacy) (17). In order to confirm the explanatory power of HAPA in health outcomes of T2DM patients, some studies have been carried out in various countries (18). These studies have shown various results in this area. However before using a health behavior change model in educational interventions, it is necessary to test this theoretical framework in a target population to identify factors that can be handled in order to achieve optimal behavior change (14). This study aims to test the efficacy of the HAPA in predicting HD of T2DM patients due to its application for educational interventions in an Iranian population.

\section{Material and Methods}

\subsection{Procedure}

In order to test the HAPA in an Iranian diabetic population with respect to HD, a cross-sectional study was carried out in Isfahan, Iran between July and December 2015. Participants were recruited from the Al-Zahra and Feiz hospitals by convenience sampling method (data collected from patients who were available to participate in study). The inclusion criteria were the development of T2DM and no history of T2DM complications (e.g. foot ulcer, diabetic retinopathy). The exclusion criteria were mental and disabling disorders according to files in their medical records (e.g. depression, motor problems). Also, those who had been converted to patients by complications of diabetes were excluded from the study. Participants were referred to the researchers through nurses after the goal of the study was explained to them. Before filling the questionnaire, the primary researcher briefly explained the purpose of the study to the participants. For those participants who were unable to fill the questionnaire, data were collected by trained interviewers through face-to-face interview. Based on experts' recommendations, collecting the sample size of 5 to 10 for each parameter in model was appropriate in order to run a structural equations modeling (19). Since there were 35 parameters in our model, the number of 250 participants seemed to be adequate.

\subsection{Inventory}

The questionnaire of this study included seven construct of HAPA: outcome expectancies ( 7 items ranged from 7 to 49 scores), risk perception ( 3 items ranged from 3 to 21 scores), action self-efficacy ( 4 items ranged from 4 to 28 scores), Intention ( 3 items ranged from 3 to 21 scores), action and coping planning (5 items ranged from 5 to 35 scores), maintenance self-efficacy (4 items ranged from 4 to 28 scores) and recovery self-efficacy (4 items ranged from 4 to 28 scores). All HAPA inventory scales were scored from 1 to 7 where higher scores indicate better status of responder in that scale. Internal consistency was assessed by Cronbach's alpha coefficient. The validity and reliability of this questionnaire has been previously tested by Rohani et al. (20).

\subsection{Measures}

\subsubsection{Risk perceptions:}

Participant perceived risk on heart attack; stroke and cardiovascular disease were assessed. In order to evaluate the perceived absolute own risk, participants were asked to estimate the chance of facing each health problem, for instance, "How high do you think your risk of heart attack is during your life time?" (21). Participants rated their odds of developing each disorder in the future using a separate seven-point scale ( $1=$ very unlikely; $4=$ moderately likely; $7=$ very likely) (22).

2.3.2. Outcome expectancies:

Outcome expectancies were measured by seven items based on Ajzen's recommendations (23) and participant's feedbacks. They were requested: "What do you think will be the consequences for yourself if you adopt an HD?" 
Following this header, responses were reduced to seven more specific questions and were identified based on previous research among the patients with type 2 diabetes $(24,25)$ such as: "If I stick to a low-fat diet, then ..." (a) "I will be healthier," (b) "I will feel better mentally," (c) "It will improve my body weight. Participants stated their compromise with the anchors of each pair using a seven-point scale 1 (strongly disagree) to 7 (strongly agree).

2.3.3. Task self-efficacy:

To assess the perceived action self-efficacy, we used four different items based on Schwarzer's recommendations (26). The following stem for all items was used, "How sure are you that you can overcome the following obstacles? I can start an HD even... " Afterwards, specific barriers were presented such as: (a) "if I initially have to make plans," (b) "if it takes a long time to get used to it," (c) "if I have to start all over again several times until I succeed." Responses were made on 7-point scales ranging from 1 (strongly disagree) to 7 (strongly agree).

\subsubsection{Behavioral intention:}

For assessment of intention to follow an HD, 3 items were applied adapted from Ajzen (23): (1) "I intend to follow an HD each day in the next 2 months," with responses from 1 (extremely unlikely) to 7 (extremely likely); (2) "I will try to follow an HD each day in the next 2 months," with responses from 1 (definitely false) to 7 (definitely true); and (3) "I plan to follow an HD each day in the next 2 months," with responses from 1 (strongly disagree) to 7 (strongly agree).

\subsubsection{Action and coping planning:}

Action planning was assessed with two items recommended by Schwarzer (16). Participants rated 1 (strongly disagree) to 7 (strongly agree) whether they had made detailed plans regarding their HD in terms of: (a) how; and (b) when they will start an HD. Three items, with the same scale anchors as action planning, were used to assess this concept. Participants scored if they had made plans in detail about: (a) what to do if their plans got into trouble; (b) how to face the defeats in their plans; and (c) how to stick with their aims even under difficult circumstances. These items were made based on Schwarzer's recommendations (16).

\subsubsection{Maintenance self-efficacy:}

Maintenance self-efficacy measured confidence of individuals in their ability to continue an HD even if they were blocked by some barriers. We identified four barriers from previous research within the type 2 diabetes patients (27, 28). Time limitation for cooking, the taste of foods and peer pressure were some of these barriers. A seven-point scale $[1=$ not confident at all and $7=$ completely confident $]$ was used to rate the items.

2.3.7. Recovery self-efficacy:

Recovery self-efficacy measured participants' convictions to see if they could recover themselves after initially failing (16). Participants were asked to answer the following questions regarding their confidence in being able to return to an HD after quitting this behavior using a seven-point scale as above: "I am sure I can continue a healthy diet (a) "if I have sometimes not succeeded in doing so," (b) "if I have not eaten healthy foods for a day," (c) "if I have not eaten healthy foods for a week," (d) "if I have not eaten healthy foods for a month,".

\subsubsection{Healthful diet behavior:}

Participants self-reported this factor using the nutrition style questionnaire designed by Renner and Schwarzer (29) with 17 questions related to HD such as: (a) "I follow a low-fat diet", (b) "When I eat dairy products or drink milk, I choose low-fat products (such as low-fat milk or yogurt)", (c) "I usually eat fresh food" and (d) "I only eat low-salt food" with a 4-point scale ranging from 1 (strongly disagree) to 4 (strongly agree). Averaging on questions, the range of 17 to 68 was gained with higher scores indicating healthier nutrition style.

\subsection{Ethical Considerations}

The experiment was approved by the ethics committee of Isfahan University of Medical Sciences No. 394097. All the participants were assured that their information would be kept with confidence remaining under the custody of the main researcher and would not be available to any unauthorized person except supervisors.

\subsection{Data Analysis}

Descriptive statistics were calculated for all variables. For missing data, the median score for each person on each question was imputed so that it would have a value and not be biased at a lower score. Structural equation modeling (SEM) in AMOS 21 using Maximum Likelihood (ML) estimation was used to test the hypothesis. The overall model fit was evaluated using chi-square (CMIN) and relative chi-square (CMIN/df), comparative fit index (CFI), normed fit index (NFI), root mean square error of approximation (RMSEA). The following values indicate good fit of the model to the data: CMIN/df between 1 and 2, CFI and NFI ranging from .90 to 1, RMSEA below .08 (19).

\section{Results}

The first 243 participants were referred to the study according to inclusion criteria. Based on exclusion criteria, 40 participants dropped out of the study. Out of 203 T2DM patients who completed the questionnaire, 117 were male 
and 86 were female. The mean age of participants was 51.8 years $(\mathrm{SD}=12.8)$ with average of 8.5 years $(\mathrm{SD}=6.6)$ for their duration of disease. The other demographic characteristics are represented in Table 1. After calculating the score of each construct by summing up the corresponding questions, mean score for each one was obtained. Cronbach's alpha for all scales was almost in an appropriate range (Table 2). Calculated face, content and criterion validity were also acceptable (20). The results of the structural model and fit indices were as follows: X2=1.99 $(\mathrm{p}<0.001), \mathrm{RMSEA}=0.082, \mathrm{CFI}=0.903$, NFI $=0.899$, indicating a satisfactory fit. However, except for RMSEA, others could not reach the acceptable threshold, which, in our opinion, is due to insufficiency of the sample size that is regarded as a limitation in this study. As a result, the HAPA model is reasonably predicting HD. All coefficients were statistically significant $(\mathrm{p}<0.05)$, except for the paths from risk perception to behavioral intention and from maintenance self-efficacy to HD behavior. The estimates of the coefficients and corresponded p-values are displayed on Figure 1.

\subsection{Predictors of intention}

Behavioral intention was associated with action self-efficacy $(\beta=2.49, \mathrm{p}<0.001)$ and outcome expectancy $(\beta=-1.92$, $\mathrm{p}<0.001)$. That is, nutrition behavior is increased by higher action self-efficacy and lower outcome expectancy. Risk perception did not predict intention to participate in nutrition behavior $(\beta=-0.56, p=0.073)$.

\subsection{Predictors of planning}

Behavioral intention $(\beta=1.18, p<0.001)$ and maintenance self-efficacy $(\beta=0.61, p<0.001)$ were positively associated with action and coping planning. That is, the greater the behavioral intention and maintenance self-efficacy, the better the planning.

\subsection{Predictors of behavior}

Finally, HD was significantly predicted by planning and recovery self-efficacy so that higher scores on action and coping planning $(\beta=5.36, p=0.027)$, and recovery self-efficacy $(\beta=5.67, p=0.021)$ were associated with higher scores on nutrition behavior. No statistical association was found between maintenance self-efficacy and behavior.

Table 1. Demographic and health characteristics of participants $(n=203)$

\begin{tabular}{|l|l|l|l|}
\hline Variable & Mean (SD) & $\mathrm{n}(\%)$ \\
\hline Age & Male & $51.8(16.1)$ & \\
\hline \multirow{2}{*}{ Gender } & Female & & $117(57.6)$ \\
\hline \multirow{3}{*}{ Education } & High school or less & & $86(42.3)$ \\
\hline & Under graduate & & $171(84.2)$ \\
\hline & Post graduate & & $29(14.3)$ \\
\hline \multirow{3}{*}{ Marital status } & Single & & $3(1.5)$ \\
\hline & Married & $17(8.3)$ \\
\hline & Divorced & & $167(82.2)$ \\
\hline & Widowed & $6(2.9)$ \\
\hline \multirow{5}{*}{ Income adequacy } & Adequate & & $13(6.4)$ \\
\hline & Not adequate & & $33(16.3)$ \\
\hline Family history of diabetes & Yes & $170(83.7)$ \\
\hline & No & & $55(27.2)$ \\
\hline Diabetes duration (year) & & & $148(72.8)$ \\
\hline Healthful diet score (17-68) & & $8.5(6.6)$ & \\
\hline
\end{tabular}

Table 2. Summary of HAPA constructs

\begin{tabular}{|l|l|l|l|}
\hline Construct (Range) & No of items & Mean (SD) & Cronbach's alpha \\
\hline Risk perception (3-21) & 3 & $15.02(2.85)$ & 0.90 \\
\hline Action self-efficacy (4-28) & 4 & $14.85(4.91)$ & 0.95 \\
\hline Outcome expectation (7- 49) & 7 & $34.21(4.39)$ & 0.85 \\
\hline Behavioral intention (3-21) & 3 & $12.70(3.51)$ & 0.69 \\
\hline Action and coping planning (5-35) & 5 & $19.90(5.59)$ & 0.94 \\
\hline Maintenance self-efficacy (4-28) & 4 & $14.82(4.08)$ & 0.73 \\
\hline Recovery self-efficacy (4-28) & 4 & $15.37(4.45)$ & 0.92 \\
\hline
\end{tabular}




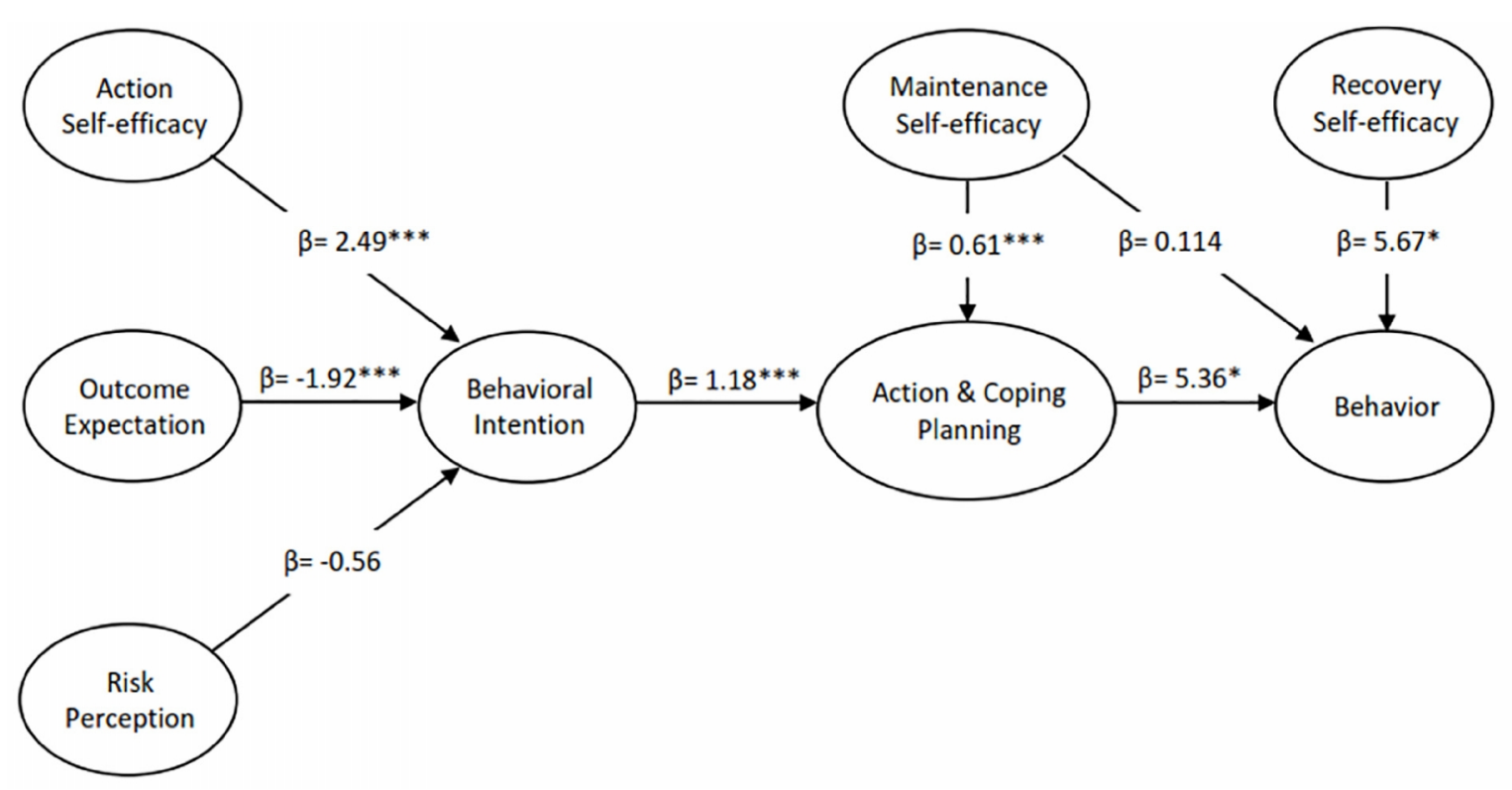

Figure 1. Structural model predicting nutrition behavior using the HAPA model. Betas are standardized coefficient of the relationship between constructs. ${ }^{*} \mathrm{p}<0.05,{ }^{* * *} \mathrm{p}<0.001$

\section{Discussion}

This study was the first to apply the HAPA model to predict HD among Iranian individuals with T2DM. Overall, the model fitted well to predicting intention to HD in T2DM patients; similar to previous prediction studies of dietary behaviors using the HAPA (30-32). Successful replication of the HAPA in Iranian T2DM patients has been the first step in the present analysis. This replication adds to the generalizability of the model since previous studies were mainly conducted with other populations with different cultures. The current study found that action self-efficacy was a significant predictor of HD Similar to our study, Mullan et al. (33) found action self-efficacy was the most significant predictor of dietary behaviors intention, indicating when promoting dietary behaviors among persons who are in intentional phase, interventions should include details that aim to build action self-efficacy such as having peer model methods or providing situations for beginners to try healthy foods leading to creation of potentials for mastery experiences (34). For people who have moved into the volitional phase, purpose of the interventions should be maintenance self-efficacy and recovery self-efficacy, as our study findings indicate that maintenance self-efficacy and recovery self-efficacy are predictors of dietary behavior. The same finding has been represented in the other studies on health behaviors such as physical activity $(31,35)$. Furthermore, building multiple types of self-efficacy can help individuals maintain their HD. While a number of the HAPA tenets $(15,17)$ were supported in the current study, there were some inconsistencies from predicted relationships. Outcome expectations had an inverse association with intentions to adopt an HD. This is in line $(35,36)$ and opposite $(17,31)$ to some previous researches that have been demonstrated in other studies. In our opinion, the latter could be due to low life expectancy within diabetes patients. With this in mind, further studies are recommended to be carried out since there are paradoxes in this context. Similar to other studies (37), no significant relationship was found between risk perception and the intention to eat a healthful diet. It might be due to the fact that the fear appeal approach has focused on using risk communication to let people realize to what extent they are at risk of illness or injury. The effectiveness of such interventions as stand-alone-strategies is doubtful at the least (38). On the other hand, this nonsignificant link between risk perception and intention might be due to the differences in the way of measuring the factors; since risk perception was generally measured whereas intention was measured in behavior-specific. Further research is needed to be carried out on these findings. The findings may have some conceptual applications for health promotion interventions, such as planning, in order to improve dietary behaviors. Several RCT's have provided enough evidence in favor of such planning interventions in the context of dietary changes (39-41). More exactly, adding planning components to interventions has led to larger effects than interventions based solely on information provision (32) which can be considered during future research.

\section{Limitations}

There are some limitations to this study. First, Evaluations were self-reported, and dietary behavior was measured retrospectively which might have been vulnerable to unintentional misreporting (42). This limitation could be 
overcome by using on-going dietary examinations such as food diaries, where individuals record details of their foods at the time of consumption or shortly afterwards. The second limitation is the cross-sectional type of design, which is based on simple association and is not based on causal relationships. Future studies might want to recheck the findings of this study by an experimental design in order to clarify causal directions. Third limitation is the shortage of sample size, which is because of low prevalence of T2DM (4-4.5\%) in the Iranian population (43).

\section{Conclusions}

Overall, our findings provide preliminary support for the practical usage of the HAPA model for predicting HD intention and behavior among patients with T2DM. These findings can be useful for guiding educational interventions with respect to HD of T2DM patients. We suggest conducting further research in this area. Using a more objective measure of HD behavior will provide extra support for this model within these patients. Finally, it should be noted that conducting randomized controlled trials in the field of dietary behaviors of T2DM patients based on the HAPA model, should be performed in the future in order to expand causality of this model.

\section{Acknowledgments:}

The authors thank Mehdi Rae for contributing in data analysis. This article was derived from a PhD thesis research project carried out in Isfahan University of Medical Sciences. Research project number: 394097.

\section{Conflict of Interest:}

There is no conflict of interest to be declared.

\section{Authors' contributions:}

All authors contributed to this project and article equally. All authors read and approved the final manuscript.

\section{References:}

1) Aguiar EJ, Morgan PJ, Collins CE, Plotnikoff RC, Callister R. Efficacy of interventions that include diet, aerobic and resistance training components for type 2 diabetes prevention: a systematic review with metaanalysis. Int J Behav Nutr Phys Act. 2014; 15(11): 2. doi: 10.1186/1479-5868-11-2. PMID: 24423095, PMCID: PMC3898566.

2) Dunkley AJ, Bodicoat DH, Greaves CJ, Russell C, Yates T, Davies MJ, et al. Diabetes prevention in the real world: effectiveness of pragmatic lifestyle interventions for the prevention of type 2 diabetes and of the impact of adherence to guideline recommendations: a systematic review and meta-analysis. Diabetes Care. 2014; 37(4): 922-33. doi: 10.2337/dc13-2195. PMID: 24652723.

3) Esteghamati A, Etemad K, Koohpayehzadeh J, Abbasi M, Meysamie A, Noshad S, et al. Trends in the prevalence of diabetes and impaired fasting glucose in association with obesity in Iran: 2005-2011. Diabetes Res Clin Pract. 2014; 103(2): 319-27. doi: 10.1016/j.diabres.2013.12.034. PMID: 24447808.

4) Ebrahimi H, Emamian MH, Shariati M, Hashemi H, Fotouhi A. Diabetes mellitus and its risk factors among a middle-aged population of Iran, a population-based study. International Journal of Diabetes in Developing Countries. 2015: 36(2): 1-8.

5) Meraci M, Feizi A, Bagher NM. Investigating the prevalence of high blood pressure, type 2 diabetes mellitus and related risk factors according to a large general study in Isfahan-using multivariate logistic regression model. Health System Research. 2012: 8(2).

6) Palermo A, Maggi D, Maurizi AR, Pozzilli P, Buzzetti R. Prevention of type 2 diabetes mellitus: is it feasible? Diabetes Metab Res Rev. 2014; 30 Suppl 1: 4-12. doi: 10.1002/dmrr.2513. PMID: 24353270.

7) Chasan-Taber L. Lifestyle interventions to reduce risk of diabetes among women with prior gestational diabetes mellitus. Best Pract Res Clin Obstet Gynaecol. 2015; 29(1): 110-22. doi: 10.1016/j.bpobgyn.2014.04.019. PMID: 25220104 PMCID: PMC4282816.

8) Haw JS, Galaviz KI, Straus AN, Kowalski AJ, Magee MJ, Weber MB, et al. Long-term Sustainability of Diabetes Prevention Approaches: A Systematic Review and Meta-analysis of Randomized Clinical Trials. JAMA Intern Med. 2017; 177(12): 1808-17. doi: 10.1001/jamainternmed.2017.6040. PMID: 29114778 PMCID: PMC5820728.

9) Shrestha A, Karmacharya BM, Khudyakov P, Weber MB, Spiegelman D. Dietary Interventions to Prevent and Manage Diabetes in Worksite Settings: a Meta-Analysis. J Occup Health. 2018; 60(1): 31-45. doi: 10.1539/joh.17-0121-RA. PMID: 29187673 PMCID: PMC5799099. 
10) Blue CL, Marrero DG, Black DR. Physical activity belief scales for diabetes risk: development and psychometric testing. Health Educ Behav. 2007; 35(3): 316-31. doi: 10.1177/1090198106297060. PMID: 17620669.

11) Hankonen N, Sutton S, Prevost AT, Simmons RK, Griffin SJ, Kinmonth AL, et al. Which Behavior Change Techniques are Associated with Changes in Physical Activity, Diet and Body Mass Index in People with Recently Diagnosed Diabetes? Ann Behav Med. 2015: 49(1): 1-11. doi: 10.1007/s12160-014-9624-9. PMID: 24806469 PMCID: PMC4335098.

12) Gaston A, Prapavessis H. Using a combined protection motivation theory and health action process approach intervention to promote exercise during pregnancy. J Behav Med. 2014: 37(2): 1-12. doi: 10.1007/s10865-012-9477-2. PMID: 23180287.

13) Payaprom Y, Bennett P, Alabaster E, Tantipong H. Using the Health Action Process Approach and implementation intentions to increase flu vaccine uptake in high risk Thai individuals: A controlled beforeafter trial. Health Psychol. 2011; 30(4): 492-500. doi: 10.1037/a0023580. PMID: 21534678.

14) Radtke T, Kaklamanou D, Scholz U, Hornung R, Armitage CJ. Are Diet-Specific Compensatory Health Beliefs Predictive of Dieting Intentions and Behaviour? Appetite. 2014. doi: 10.1016/j.appet.2014.01.014. PMID: 24472827.

15) Schwarzer R. Modeling health behavior change: How to predict and modify the adoption and maintenance of health behaviors. Applied Psychology. 2008; 57(1): 1-29. doi: 10.1111/j.1464-0597.2007.00325.x.

16) Schwarzer R, Sniehotta FF, Lippke S, Luszczynska A, Scholz U, Schüz B, et al. On the assessment and analysis of variables in the health action process approach: Conducting an investigation. Berlin: Freie Universeitat Berlin; 2003.

17) Schwarzer R, Lippke S, Luszczynska A. Mechanisms of health behavior change in persons with chronic illness or disability: the Health Action Process Approach (HAPA). Rehabil Psychol. 2011; 56(3): $161-70$. doi: 10.1037/a0024509. PMID: 21767036.

18) MacPhail M, Mullan B, Sharpe L, MacCann C, Todd J. Using the health action process approach to predict and improve health outcomes in individuals with type 2 diabetes mellitus. Diabetes Metab Syndr Obes. 2014; 7: 469-79. doi: 10.2147/DMSO.S68428. PMID: 25342914 PMCID: PMC4206248.

19) Brown TA. Confirmatory factor analysis for applied research. Guilford Publications; 2015.

20) Rohani H, Eslami AA, Ghaderi A, Bidkhori M, Raei M. Development and psychometric evaluation of a health action process approach inventory for healthful diet among Type 2 diabetes patients. Int $\mathrm{J}$ Prev Med. 2016; 7(1): 69. doi: 10.4103/2008-7802.181333. PMID: 27195101 PMCID: PMC4863400.

21) Perloff LS, Fetzer BK. Self-other judgments and perceived vulnerability to victimization. Journal of Personality and social Psychology. 1986; 50(3): 502. doi: 10.1037/0022-3514.50.3.502.

22) Renner B, Knoll N, Schwarzer R. Age and body make a difference in optimistic health beliefs and nutrition behaviors. International Journal of Behavioral Medicine. 2000; 7(2): 143-59. doi: 10.1207/S15327558IJBM0702 4.

23) Ajzen I. The theory of planned behavior. Organizational behavior and human decision processes. 1991; 50(2): 179-211. doi: 10.1016/0749-5978(91)90020-T.

24) Suri B, Tariq O. General Perception of Diabetes, Social Support and Outcome Expectancies Related to Adherence Among People With Type 2 Diabetes. European Health Psychologist. 2014; 16(S): 348.

25) Chlebowy DO, Garvin BJ. Social Support, Self-efficacy, and Outcome Expectations Impact on Self-care Behaviors and Glycemic Control in Caucasian and African American Adults With Type 2 Diabetes. Diabetes Educ. 2006; 32(5): 777-86. doi: 10.1177/0145721706291760.

26) Schwarzer R, Renner B. Social-cognitive predictors of health behavior: action self-efficacy and coping selfefficacy. Health Psychol. 2000; 19(5): 487-95. doi: 10.1037/0278-6133.19.5.487. PMID: 11007157.

27) Ebrahim Z, De Villiers A, Ahmed T. Factors influencing adherence to dietary guidelines: a qualitative study on the experiences of patients with type 2 diabetes attending a clinic in Cape Town. Journal of Endocrinology, Metabolism and Diabetes of South Africa. 2014; 19(2): 76-84. doi: 10.1080/16089677.2014.11073604.

28) Vijan S, Stuart N, Fitzgerald J, Ronis D, Hayward R, Slater S, et al. Barriers to following dietary recommendations in Type 2 diabetes. Diabet Med. 2005; 22(1): 32-8. doi: 10.1111/j.14645491.2004.01342.x.

29) Sonia L, Jochen PZ. Understanding and Modeling Health Behavior: The Multi-Stage Model of Health Behavior Change. J Health Psychol. 2006; 11(1): 37-50. doi: 10.1177/1359105306058845. 
30) Godinho CA, Alvarez MJ, Lima ML, Schwarzer R. Health messages to promote fruit and vegetable consumption at different stages: A match-mismatch design. Psychol Health. 2015; 30(12): 1410-32. doi: 10.1080/08870446.2015.1054827. PMID: 26010304.

31) Lippke S, Plotnikoff RC. Testing two principles of the Health Action Process Approach in individuals with type 2 diabetes. Health Psychol. 2014; 33(1): 77. doi: 10.1037/a0030182.

32) Zhou G, Gan Y, Knoll N, Schwarzer R. Proactive coping moderates the dietary intention-planningbehavior path. Appetite. 2013; 70: 127-33. doi: 10.1016/j.appet.2013.06.097. PMID: 23856434.

33) Mullan BA, Wong CL, O'Moore K. Predicting hygienic food handling behaviour: modelling the health action process approach. British Food Journal. 2010; 112(11): 1216-29. doi: 10.1108/00070701011088205.

34) Bandura A. Self-efficacy mechanism in human agency. American psychologist. 1982; 37(2): 122. doi: 10.1037/0003-066X.37.2.122.

35) Perrier MJ, Sweet SN, Strachan SM, Latimer-Cheung AE. I act, therefore I am: Athletic identity and the health action process approach predict sport participation among individuals with acquired physical disabilities. Psychology of Sport and Exercise. 2012; 13(6): 713-20. doi: 10.1016/j.psychsport.2012.04.011.

36) Scholz U, Keller R, Perren S. Predicting behavioral intentions and physical exercise: A test of the health action process approach at the intrapersonal level. Health Psychol. 2009; 28(6): 702 . doi: $10.1037 / \mathrm{a} 0016088$.

37) Scholz U, Ochsner S, Hornung R, Knoll N. Does Social Support Really Help to Eat a Low - Fat Diet? Main Effects and Gender Differences of Received Social Support within the Health Action Process Approach. Appl Psychol Health Well Being. 2013; 5(2): 270-90. doi: 10.1111/aphw.12010. PMID: 23625820.

38) Calisir F, Lehto MR. Young drivers' decision making and safety belt use. Accid Anal Prev. 2002; 34(6): 793-805. PMID: 12371784.

39) Gholami M, Lange D, Luszczynska A, Knoll N, Schwarzer R. A dietary planning intervention increases fruit consumption in Iranian women. Appetite. 2013; 63: 1-6. doi: 10.1016/j.appet.2012.12.005. PMID: 23266280.

40) Guillaumie L, Godin G, Manderscheid JC, Spitz E, Muller L. Self-efficacy and implementation intentionsbased interventions on fruit and vegetable intake among adults: impact at 12-month follow-up. Glob Health Promot. 2013; 20(2 suppl): 83-7. doi: 10.1177/1757975913483336. PMID: 23678501.

41) Wiedemann AU, Lippke S, Schwarzer R. Multiple plans and memory performance: Results of a randomized controlled trial targeting fruit and vegetable intake. J Behav Med. 2012; 35(4): 387-92. doi: 10.1007/s10865-011-9364-2. PMID: 21706212.

42) Hassan E. Recall bias can be a threat to retrospective and prospective research designs. The Internet Journal of Epidemiology. 2006; 3(2): 339-412.

43) Lotfi MH, Saadati H, Afzali M. Prevalence of diabetes in people aged $\geqslant 30$ years: the results of screening program of Yazd Province, Iran, in 2012. J Res Health Sci. 2013; 14(1): 88-92. PMID: 24402857. 